\section{Low frequency 3D synthetic aperture radar for the remote intelligence of building interiors}

\section{Andre, B. Faulkner and M. Finnis}

Low Frequency (LF) Synthetic Aperture Radar (SAR) images offer a viable approach to determining the architecture and contents of buildings and underground bunkers via remote sensing. Often however, standard 2D SAR images can be difficult to interpret due to component signatures from different heights being projected into the scene leading to confused results. In this research, measurement results have shown that the full Nyquist 2D aperture scan approach to 3D through-wall LF SAR, provides focussed 3D resolution of a wall and contents behind it in a number of frequency bands. Full scale radar system upgrades are ongoing in order to investigate numerous other scenarios, however in the meantime, sparse 2D aperture scanning investigations have been undertaken with a prototype radar scanner. Whilst this kind of collection cannot achieve the low sidelobe levels of full Nyquist 2D aperture collections, these prototype scanner measurements are much faster to collect, and have shown encouraging results of sufficient image quality to determine the $3 \mathrm{D}$ configuration of prominent features in the target scene, albeit with higher sidelobe or image artefact levels.

Introduction: Low Frequency (LF) radar image formation offers a viable approach to determining the architecture and contents of buildings and underground bunkers via remote sensing. Often however, standard two dimensional images can be difficult to interpret due to component signatures from different heights of the building structure and contents being projected into the scene.

The reported work details investigations into the formation of through-wall 3D or volumetric Synthetic Aperture Radar (SAR) images, conducted with the Cranfield University Ground-Based SAR (GBSAR) system [1].

The following section details a full Nyquist and full scale 2D aperture scan measurement, and the section after describes sparse aperture 3D SAR measurements conducted with a prototype scanner, with the aim of demonstrating a more practical volumetric SAR image collection method.

Full 2D aperture $L F 3 D$ SAR scan: In order to form initial 3D radar images through a wall, a hand wound radar interferometry mount was re-purposed to enable aligned 2D SAR aperture collections and 3D SAR image formation. The interferometer mount is shown in Fig. 1 together with the broadband antenna horns employed for the experiment. The horn frequency range is $1-18 \mathrm{GHz}$, providing a potential bandwidth of $17 \mathrm{GHz}$. The horn shows relatively little beam pattern variation over the wide frequency range, allowing better quality wide-band SAR images.

The radar-near-side measured scene can be seen in Fig. 2, where a $25 \mathrm{~cm}$ trihedral is seen on a stool, and a $15 \mathrm{~cm}$ trihedral is on the ground. A large $60 \mathrm{~cm}$ trihedral is located on the radar-far-side of the wall, pictured in Fig. 3.

The wall was determined to be made of concrete breeze blocks, constituted by two layers of the blocks, together achieving a thickness of $25 \mathrm{~cm}$. The radar wall attenuation was observed to vary with frequency, with more attenuation at the higher frequencies, however in the measured frequency range of $1-5 \mathrm{GHz}$ on average the attenuation was approximately $35 \mathrm{~dB}$.

Multiple horizontal cross-range linear SAR apertures of $1.035 \mathrm{~m}$ with a spacing of $1.5 \mathrm{~cm}$ were scanned. The vertical SAR aperture consisted of 45 consecutive linear horizontal radar scans with vertical positions on the interferometer ranging over a $65.3 \mathrm{~cm}$ extent. The first 44 scans were spaced at $1.5 \mathrm{~cm}$, and the last spacing was $0.8 \mathrm{~cm}$, attaining the full extent of the interferometer.

Within the measured radar frequency range $1-5 \mathrm{GHz}$, three sub-bands were extracted for image formation: $1-3 \mathrm{GHz}, 2-4 \mathrm{GHz}$ and $3-5 \mathrm{GHz}$. These shall be referred to as sub-bands 2, 3 and 4, according to their centre frequencies in $\mathrm{GHz}$

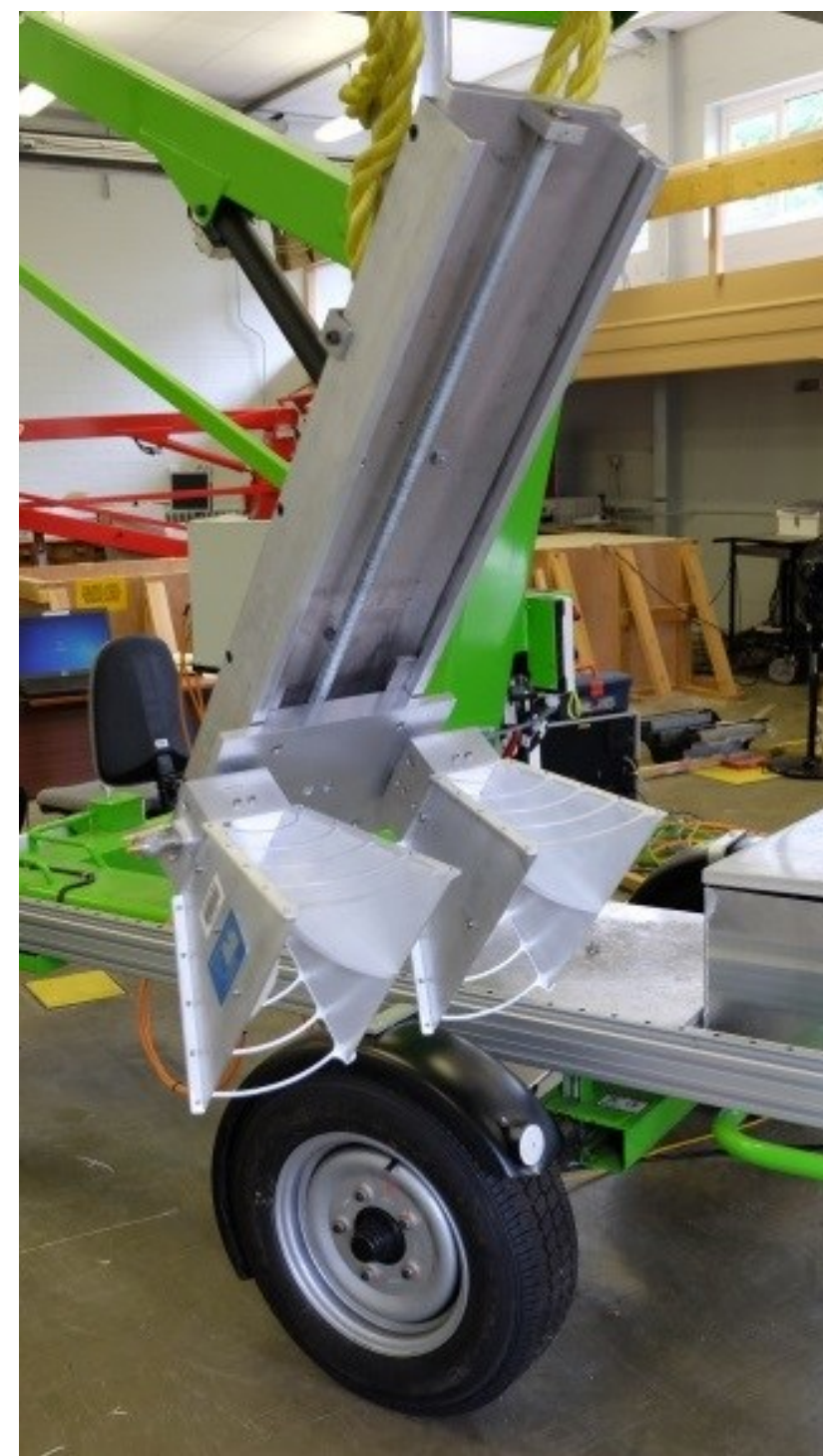

Fig. 1 Radar Interferometry mount repurposed for the collection of accurately aligned $2 D$ apertures for 3D SAR image formation. The broadband horn antennas were mounted on the interferometer handwound mechanism.

In range, the resolution obtained for each sub-band is approximately $8 \mathrm{~cm}$. In horizontal cross-range, the sub-bands $2-4$ have the approximate SAR resolutions of $22 \mathrm{~cm}, 15 \mathrm{~cm}$ and $11 \mathrm{~cm}$ respectively; in vertical cross-range the SAR resolutions are approximately $35 \mathrm{~cm}, 23 \mathrm{~cm}$ and $17 \mathrm{~cm}$ respectively.

An LF 3D volumetric SAR image was formed for each sub-band, and for the sub-bands 2-4 these are presented in Fig. 4a-c respectively. Each image is presented with a normalized intensity scale. The radar is located in the positive $y$-direction with mean coordinates relative to imaging scene centre of $(\mathrm{x}, \mathrm{y}, \mathrm{z})=(0,3.5,0.6) \mathrm{m}$, which corresponds to a ground-range of $3 \mathrm{~m}$ from the wall and height of $2.2 \mathrm{~m}$ from the ground.

The 3D SAR images were formed with the bistatic back-propagation SAR image formation algorithm $[2,3]$.

The brightest scattering centres in the images are the two trihedrals placed on the radar-near-side of wall, the smaller one being on the ground and the larger higher up on a stool. The next brightest scatterers are wall signatures, the lower one is a wall-ground multipath dihedral signature being of a horizontal elongated nature, and the higher one is a direct-wall back-scattering signature at radar height. The direct-wall signature is wider in nature as it mirrors the physical size of the 2D SAR collection aperture. The radar-far-side of wall signature is the dimmest scattering response, corresponding to the trihedral in the radaropposing room.

The three images show the varying scattering and image quality characteristics of the different scatterers, along with the variation of 
attenuation through the wall for the sub-bands 2-4. For the higher frequency sub-bands, there is better cross-range resolution, so that the radar-near-side of wall signatures are better defined with more detail. For example the direct wall signature shows disturbances due to wall fitting structures, including a pipe and electrical wall fitting. As expected, the attenuation of the radar-far-side of wall scatterer is greatest at sub-band 4, however it remained in focus in all the subbands.

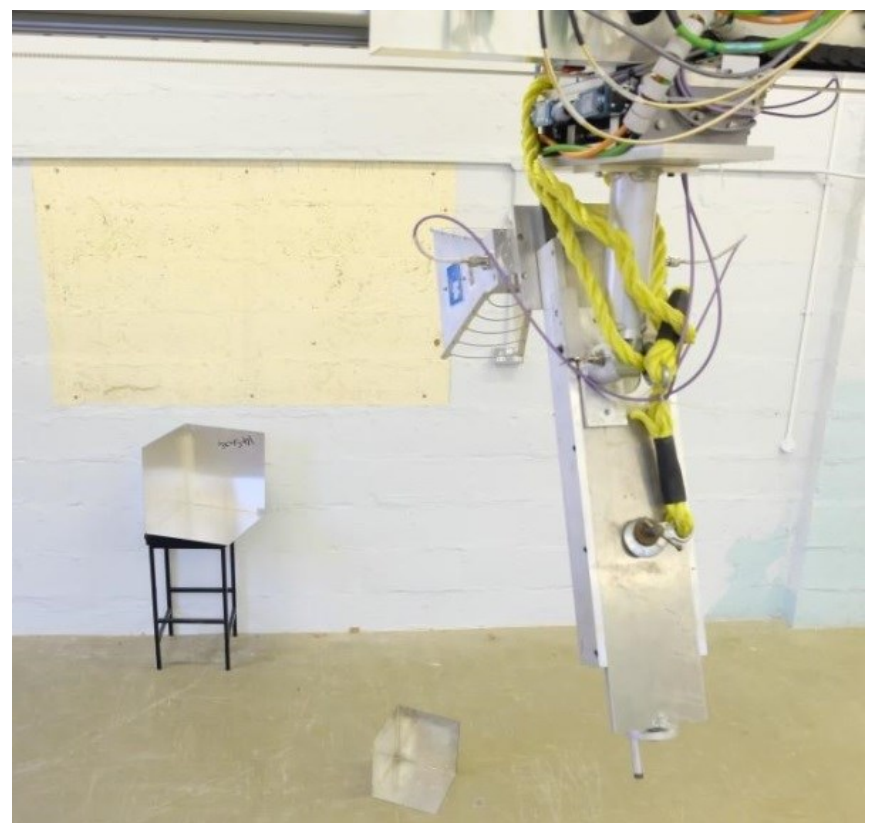

Fig. 2 Radar-near-side of concrete wall measured scene with two trihedrals and a wall present. Several pipes and electrical wall fittings are evident in the photograph. The radar horns and hand wound vertical interferometer mount are shown, with the horns at the highest setting.

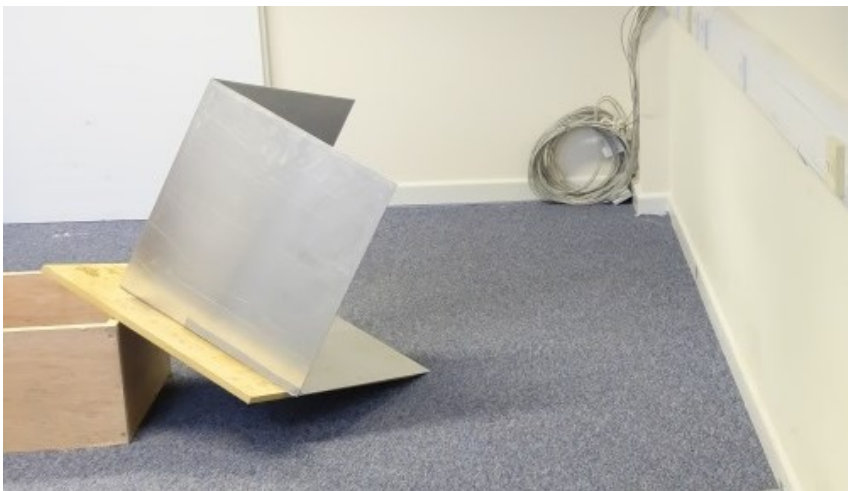

Fig. 3 Radar-far-side of concrete wall measured scene showing the large $600 \mathrm{~mm}$ trihedral target.

Whilst the full Nyquist 2D aperture SAR collection method gave high quality results, this particular measurement approach proved impractical for a full measurement campaign involving the repeated 3D SAR measurements of many different scenes with many different bistatic geometries. The reason for this being that the presented 3D SAR results required 2.5 hours of effort solely for the collection of the measured data. This level of effort was deemed excessively labour intensive.

In order to proceed further with this research, two approaches have been undertaken:

Firstly, a motorized linear vertical aperture has been purchased and is in the process of being installed on to the GB-SAR system. Together with software upgrades, this will allow automated 3D SAR collection;

Secondly, 3D SAR collection investigations have continued with a small prototype 3D scanner, with example results presented in the following section.
Other upgrades being implemented on the full-scale GBSAR system include the installation of microwave amplifiers and higher gain wideband antennas, these upgrades allowing the imaging of fainter reflecting objects, necessary for useful gathering of intelligence from the radar-far-side of wall scenes.
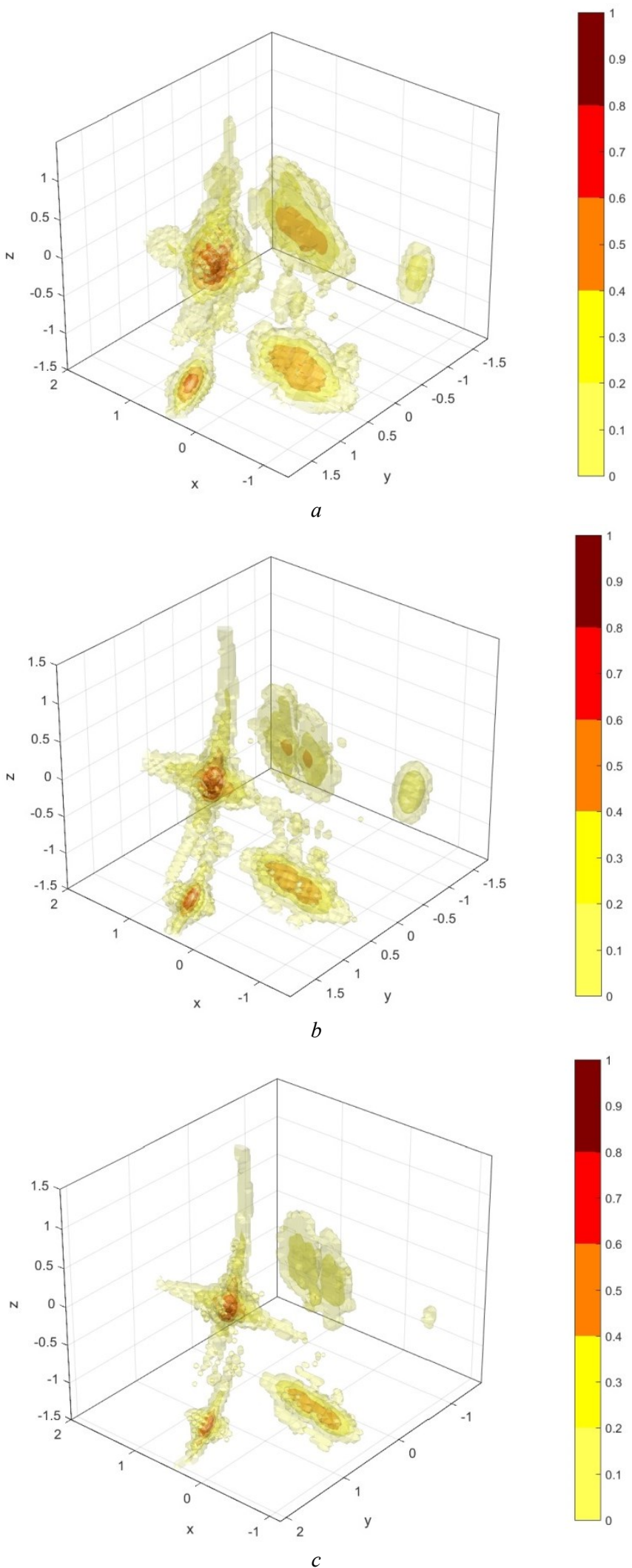

Fig. 4 Representations of a $3 D$ LF SAR volumetric through-wall dataset, composed of partially transparent iso-brightness contour surfaces. The images were formed with three frequency sub-bands with $2 \mathrm{GHz}$ bandwidth, the sub-band centre frequencies being:

a $2 \mathrm{GHz}$,

b $3 \mathrm{GHz}$,

c $4 \mathrm{GHz}$. 
Sparse aperture LF radar scan: This section details research towards efficient sparse 3D SAR collection apertures. Whilst the complete 2D SAR aperture scan discussed in the previous section will offer the best quality volumetric 3D SAR image results, this is not often practical to collect, in that a large number of horizontal scans at numerous heights need to be conducted and this is a time intensive process. A more practical approach could involve a sparse trajectory collection, even though volumetric image quality is likely to suffer as for example sidelobe levels may increase.

The full-scale GBSAR system upgrade to automated 2D aperture collections are ongoing, however in the meantime the prototype 2D aperture scanner pictured in Fig. 5 has been developed. This prototype scanner was developed with the Lego Mindstorms system [4], and allowed an initial measurements-based investigation into several types of fast sparse 2D aperture collections.

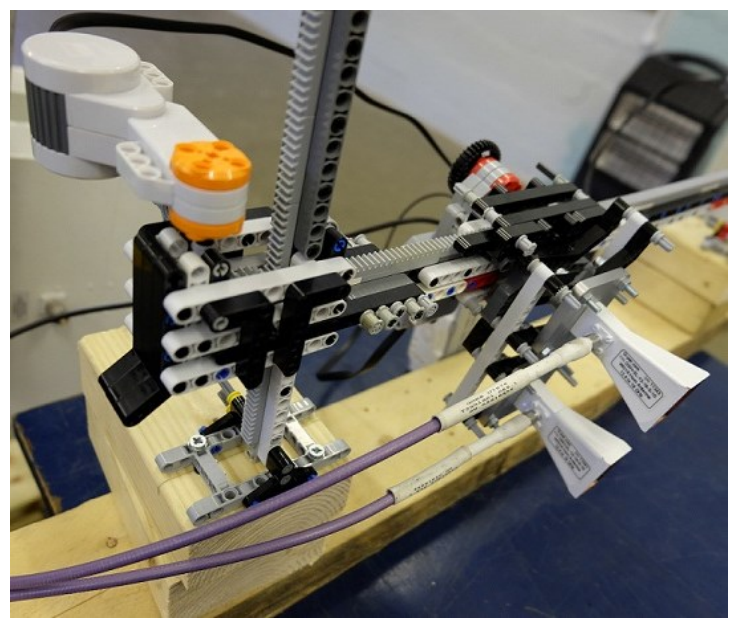

Fig. 5 Prototype $3 D$ SAR scanning system.

One such family of fast sparse collections is the Zigzag trajectory, which zigzags across the rectangular aperture a number of times: a Triple-Zigzag and Double-Zigzag trajectory are shown in Fig. $7 \mathrm{~b}$ and Fig. 7c. Note that in each figure there are overlaid Zigzag trajectories: one trajectory is for the receiver, and the other for the transmitter. The transceivers are separated in the vertical direction by $7 \mathrm{~cm}$. The corresponding full Nyquist sampled 2D aperture is shown in Fig. 7a. The figures also indicate the relative position of the target.

The target scene consists of ten $6 \mathrm{~mm}$ ball bearings at two different heights, pictured in Fig. 6. The measured frequency range was from 10$20 \mathrm{GHz}$, providing a bandwidth of $10 \mathrm{GHz}$ and a range resolution of $1.5 \mathrm{~cm}$. The horizontal and vertical apertures are $38 \mathrm{~cm}$ and $30 \mathrm{~cm}$ respectively and the cross-range resolutions achieved were approximately $2.5 \mathrm{~cm}$ at a range of $80 \mathrm{~cm}$.

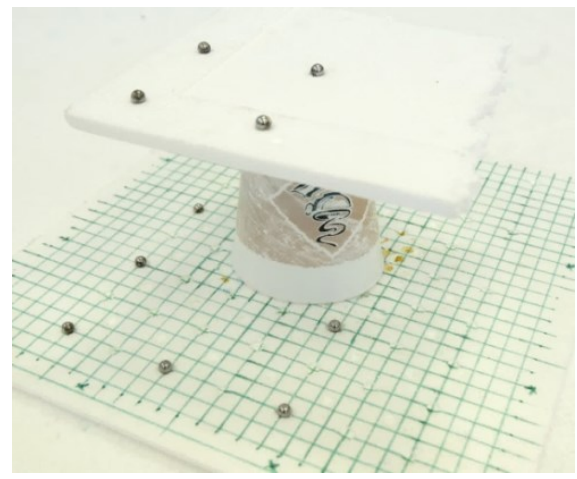

Fig. 6 Multiple scatterer target.

Projections of iso-brightness contours of the example measured volumetric SAR result are shown in Fig. 8, clearly showing the threedimensional positions of the ten ball bearings. Fig. 8a shows the full Nyquist 2D aperture collection, whereas Fig. 8b and Fig. 8c show the Triple-Zigzag and Double-Zigzag collection aperture results.
It can be seen that the full $2 \mathrm{D}$ aperture image accurately shows the position of all the ball-bearings, as does the Triple-Zigzag result. However interpretation of the Double-Zigzag is problematic as numerous artefacts have appeared as highlighted with the arrow. It is clear that the Double-Zigzag has collected insufficient information from the scene to represent it accurately.
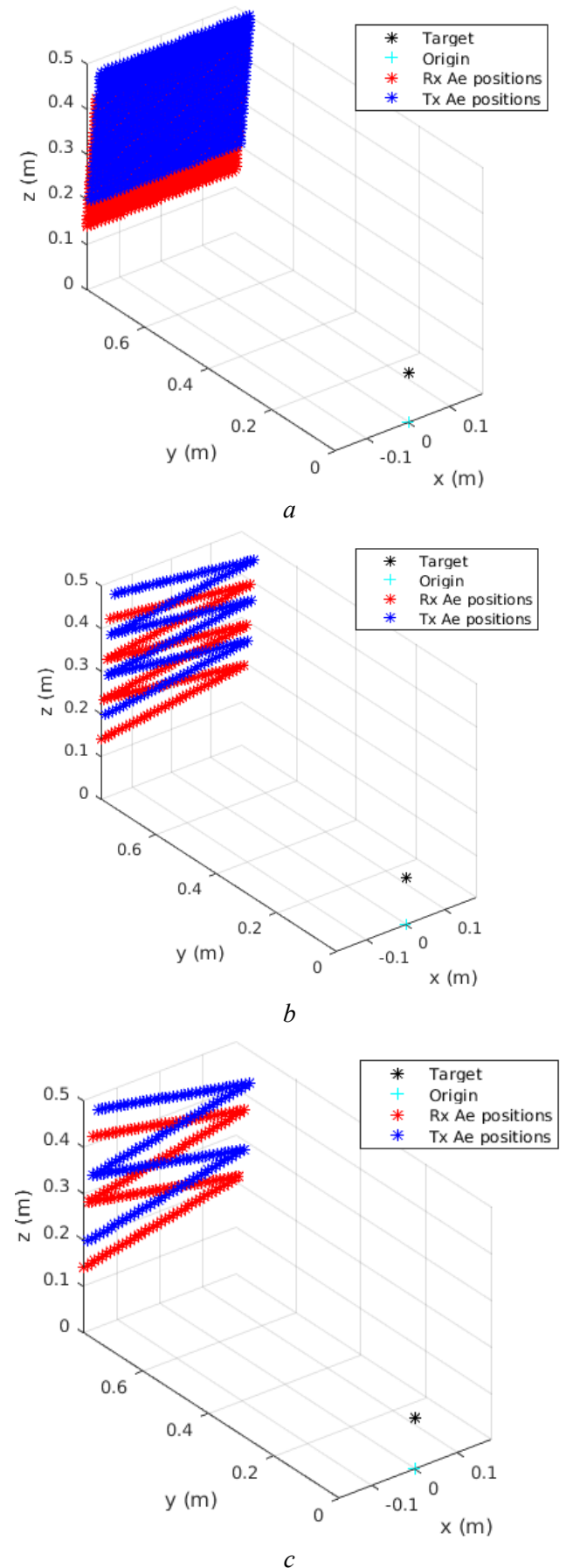

Fig. 7 Scanned transmitter and receiver horn positions and scene geometry:

a Full-Nyquist rectangular 3D SAR aperture,

$b$ Triple-Zigzag sparse 3D SAR aperture,

c Double-Zigzag sparse 3D SAR aperture.

It should be noted however, that even for the Double-Zigzag trajectory, the information collected is limited: for all renderings, only two iso-contour shells have been displayed, one for $6 \mathrm{~dB}$ below maximum brightness, and another for $12 \mathrm{~dB}$ below maximum brightness. Whilst the full $2 \mathrm{D}$ aperture collection accurately shows the location of the ball-bearings even at $12 \mathrm{~dB}$ below maximum, the TripleZigzag trajectory is full of artefacts at the lower brightness level. It can therefore be seen that sparse aperture collection trajectories do have 
their utility, but only up to a point, as they will be subject to higher sidelobe levels and artefacts in comparison to full aperture collections.
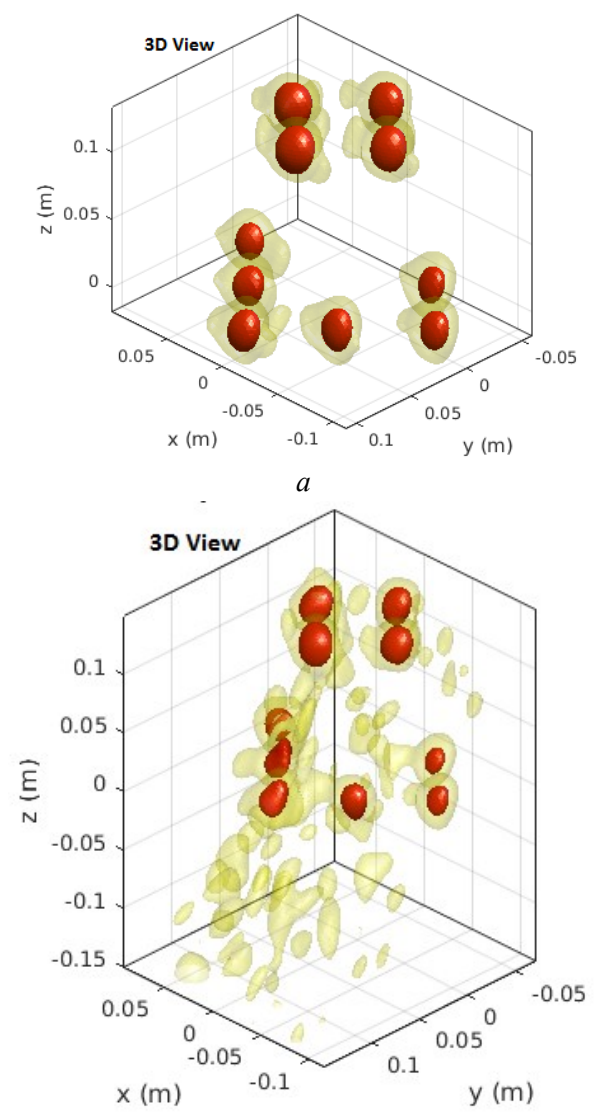

$b$

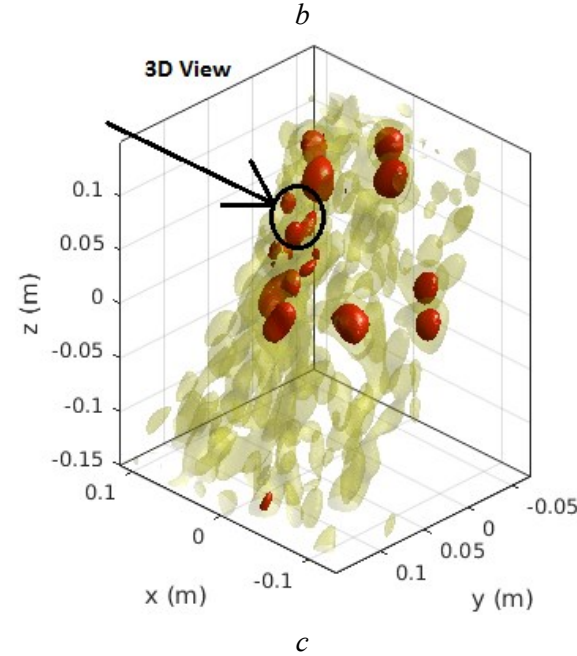

$c$

Fig. 8 Scanned Multi scatterer, 3D SAR image projections, -6dB shell: dark contours, $-12 d B$ shell: light contours, for:

a Full 2D aperture trajectory,

$b$ Triple-Zigzag sparse aperture trajectory,

c Double-Zigzag sparse aperture trajectory.

Following the methodology in [5], in order to investigate the reduction in utility of sparse aperture trajectories, repeat measurements were conducted with a single ball bearing target, to accurately determine the overall resolution at any given intensity level below maximum brightness. The measure of resolution for a given brightness level employed was the maximum Euclidian distance across the corresponding Point Spread Function (PSF) iso-brightness contour surface.

Fig. 9 shows graphs of PSF width against below maximum brightness level for four 2D aperture collection types: Full aperture, Triple-Zigzag, Double-Zigzag and Single-Zigzag. The full-aperture collection clearly performs well throughout the $12 \mathrm{~dB}$ range investigated, however it is noted that some of the other trajectories perform equally well, but only up to a point.

It can be seen that all trajectories perform equally well to the $-3 \mathrm{~dB}$ level, however below this brightness, the Single-Zigzag collection becomes rapidly unusable. The Double and Triple-Zigzag trajectories can be seen to perform as well as the full aperture scans until the $-6 \mathrm{~dB}$ and $-7 \mathrm{~dB}$ below maximum brightness levels respectively. Below these levels these sparse trajectory resolutions degrade however. Nonetheless the Double-Zigzag retains some utility until $-9 \mathrm{~dB}$ and the Triple-Zigzag throughout the whole $12 \mathrm{~dB}$ dynamic range with PSF widths below $125 \mathrm{~mm}$.

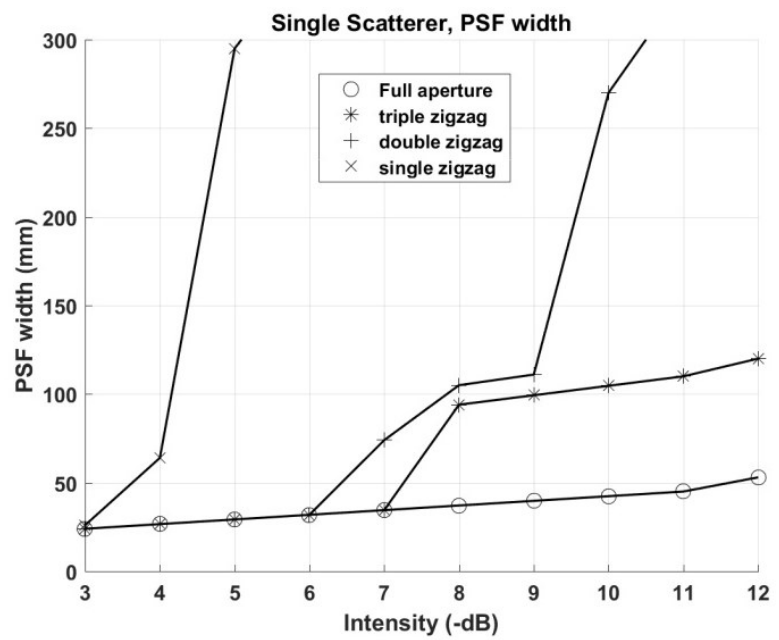

Fig. 9 PSF width versus intensity, for different sparse $3 D$ SAR aperture trajectories. The PSF width is a measure of $3 D$ SAR resolution.

Conclusion: Measurements have shown that the full 2D aperture scan approach to 3D through-wall LF SAR data collection successfully focussed a wall and contents beyond it in a number of low frequency bands. Full scale GBSAR system upgrades are ongoing in order to investigate numerous other scenarios including multistatic and multipolarimetric SAR collections with higher microwave powers.

In the meantime, sparse 2D aperture scanning investigations have been undertaken with a prototype $2 \mathrm{D}$ aperture radar scanner. Whilst this kind of collection cannot achieve the low sidelobe levels of full Nyquist 2D aperture collections, these sparse measurements are much faster to collect, and have shown encouraging results of sufficient image quality to determine the $3 \mathrm{D}$ configuration of prominent features in the target scene, albeit with higher sidelobe or image artefact brightness levels.

Acknowledgments: This work was funded by Dstl under the RIBI programme.

D. Andre (Centre for Electronic Warfare, Cyber and Information, Cranfield University), d.andre@cranfield.ac.uk

M. Finnis (Centre for Defence Engineering, Cranfield University)

B. Faulkner (Australian Army)

\section{References}

1. Andre, D., Morrison, K., Blacknell, D., Muff, D. Nottingham, Stevenson, C.: 'Very high resolution Coherent Change Detection', 2015 IEEE Radar Conference (RadarCon), May 2015, pp.1-5, DOI: 10.1109/RADAR.2016.7485116

2. Carrara, W., Goodman, R., Majewski, R.: 'Spotlight Synthetic aperture radar signal processing algorithms', Artech House, 1995, ISBN: 978-0890067284

3. Barnes, C.: 'Synthetic aperture radar, wave theory foundations, analysis and algorithms', 2014, ISBN: 978-0692313732

4. Lego: 'Mindstorms - EV3', URL: https://www.lego.com/engb/ mindstorms, 2017

5. Andre, D. "An analysis of 3D SAR from single pass nonlinear radar platform trajectories", Proceedings of SPIE, 7699, Algorithms for Synthetic Aperture Radar imagery XVII, 2010, 769908: 1-12, DOI:10.1117/12.850093 